\title{
Analysis on Crack Fatigue Growth Calculation for Nuclear Power Plant In- service Inspection
}

\author{
Cheng Yang , Xueliang Zhang and Peng Xia \\ In-service Inspection, Technical Department, Suzhou Nuclear Power Research Institute, China
}

\begin{abstract}
Fatigue is the common failure mode for nuclear mechanical components during operation which could induce crack initiation and growth. In order to predict the behaviour of fatigue crack propagating, fatigue calculation is introduced. A new method of crack fatigue growth calculation has been presented in this paper according to French RSE-M Rules. Regarding this method, detail analysis on key formulas selecting, essential calculation factors of occurrences combination, K-value calculation and mechanic modelling, characterization interval option has been performed. One fatigue crack growth calculation example of dissimilar weld of Reactor Pressure Vessel outlet nozzle to the safe end has been taken and the result demonstrates a good expectation.
\end{abstract}

\section{Introduction}

Throughout the lifetime of nuclear power plant operation, nuclear mechanical components are subject to a wide variety of loads such as pressure load, thermal load and periodic vibration load, thereby giving rise to fatigue which could induce crack initiation and growth, severely endangering the integrity of mechanical components. Therefore, during in-service inspection (ISI), once any crack has been detected through non-destructive test (NDT) which cannot be characterized by acceptance criteria, is thus required to perform crack fatigue growth calculation to predict the crack growth for the whole life of the component.

A new method of crack fatigue growth calculation has been presented in this paper according to French RSE-M Rules. Regarding this method, detail analysis on key formulas selecting, essential calculation factors of occurrences combination, K-value calculation and mechanic modelling, characterization interval option has been performed [1]. One fatigue crack calculation example of dissimilar weld of Reactor Pressure Vessel outlet nozzle to the safe end has been taken, in the hope to provide guidance on crack fatigue calculation for nuclear pressure components of M310, CPR1000, EPR and Hualong $1 \#$ reactors during ISI, or to provide reference to AP1000, Tianwan WWER-1000 reactors by ASME rules in China [2].

\section{Content of crack fatigue growth calculation}

The content of crack fatigue growth calculation is to calculate the crack initiation factor under combination occurrences. If the initiation factor is greater than 1 , the crack should be deemed initiated.

Once crack initiated, we need to calculate the size of the crack after growing within the characterization interval. After calculating, we can obtain the fracture mechanic parameters (Kcp and $J$ integral) and compare them to the specified limit values defined in RSE-M to verify the component structural integrity.

It is generally considered that the crack newly detected by NDT during ISI has initiated. Therefore, the initiation factor is not required to be calculated, so the theory of crack fatigue calculation in this paper is specific to the crack growth.

\section{Method of crack fatigue growth calculation}

The method of crack fatigue growth calculation is as follows:

(1) Calculating the stress intensity factors $K_{\mathrm{I}}, K_{\mathrm{II}}$ and $K_{\mathrm{III}}$ at the crack tip in each occurrence.

$K_{\mathrm{I}}, K_{\mathrm{II}}$ and $K_{\mathrm{III}}$ are the stress intensity factors of crack under three stress modes, which are known as opening mode, sliding mode and tearing mode. The maximum and minimum values of stress intensity factors in such three modes, i.e. $K_{\mathrm{I} \max }$ and $\mathrm{K}_{\mathrm{I} \text { min }} ; K_{\mathrm{II} \max }$ and $K_{\mathrm{II} \min } ; K_{\mathrm{III} \max }$ and $K_{\text {III min }}$ will be calculated.

(2) Calculating the equivalent stress intensity factor range $\Delta K$ and maximum stress intensity factor $K_{\max }$ for combination occurrences.

In case of combination of occurrences $\mathrm{p}$ and $\mathrm{q}, \mathrm{K}$ values of stress intensity factors in such three modes can be calculated by Eq. 1 , and then $\Delta K_{\mathrm{I}}, \Delta K_{\mathrm{II}}$ and $\Delta K_{\mathrm{III}}$ (difference between $K$ values for Occurrences $\mathrm{p}$ and $\mathrm{q}$ ) 
accordingly. This paper will calculate the equivalent stress intensity factor range and the maximum stress intensity factor using linear combination method which is simple, highly precise and easily for computer programmed, and described as follows:

$$
\Delta K=\Delta K_{\mathrm{I}}+\Delta K_{\mathrm{II}}+0.74 \Delta K_{\mathrm{III}}
$$

Taking the maximum value of $K_{\mathrm{I}}, K_{\mathrm{II}}$ and $K_{\mathrm{III}}$ for combination occurrences $\mathrm{p}$ and $\mathrm{q}$, and then the maximum stress intensity factors $K_{\max }$ and $K_{\min }$ of such combination occurrences can be calculated.

$$
\begin{gathered}
K_{\max }=\max \left(K_{\mathrm{I}}, 0\right)+\left|K_{\text {II }}\right|+\left|K_{\text {III }}\right| \\
K_{\text {min }}=K_{\text {max }}-\Delta K
\end{gathered}
$$

Regarding different combination occurrences, it can be calculated different $\Delta K, K_{\max }$ and $K_{\min }$, and then these values could be permutated to find Maximum Value $\Delta K(m, n)$ and their corresponding $K_{\max }(m, n)$, where $m$ and $n$ are the combination occurrences when $\Delta K$ is maximized [3].

(3) Calculating the corrected equivalent stress intensity factor range $\Delta K_{C P}$ for combination occurrences.

There is stress concentration at the crack tip and therefore existing plastic zone at the crack tip, which has changed the stress distribution of crack. So during calculation, the corrected equivalent stress intensity factor range $\Delta K_{C P}$ of the plastic zone should be considered. Here we describe the calculation method of $\Delta K_{C P}$, taking a finite length emerging crack as an example. Suppose the dimension size of finite length emerging crack is that: Height $(h)=a$ and Length $(l)=2 c$, as shown in Figure 1.

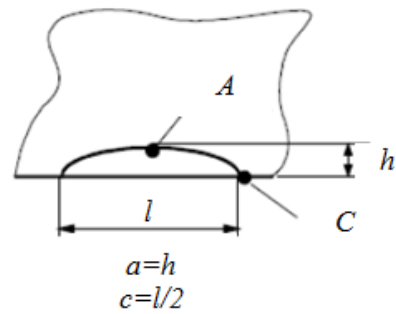

Figure 1. Dimension of finite length emerging crack.

(a) Calculating the radius of the plastic zone $r_{y A}$ :

$$
r_{y A}=\frac{1}{6 \pi}\left[\frac{\Delta K_{d}(m, n)}{S_{y A}(m)+S_{y A}(n)}\right]^{2}
$$

where $S_{y A}(m)$ and $S_{y A}(n)$ are the yield stress values of the material at the temperatures reached at Point $\mathrm{A}$, at times $m$ and $n$ respectively, which can be acquired by consulting the component design file. The radius of plastic zone at Point $\mathrm{C}$ can be calculated with the same formula above [4]0.

(b) Selecting formula :

For emerging defect, the maximum equivalent stress intensity factor $K_{\max }^{\text {meca }}(m, n)$ due to mechanical loads under the combination occurrences at Point $A$ should be calculated with the same method as $K_{\max }$, while only mechanical load is used in the process of calculation.

$$
\text { If } K_{\operatorname{maxA}}^{\mathrm{meca}}(m, n)>0.2 K_{\max }(m, n)
$$

Then $K_{c p A}=\Delta K_{A}(m, n) \sqrt{\frac{a+r_{y A}}{a}}$

$$
\Delta K_{c p C}=\Delta K_{C}(m, n) \sqrt{\frac{a+r_{y C}}{a}}
$$

Otherwise $K_{c p A}=\Delta K_{A}(m, n)$

$$
\Delta K_{c p C}=\Delta K_{C}(m, n)
$$

(4) Calculating $\Delta a$ and $\Delta c$.

Fatigue crack growth is estimated by using the corrected stress intensity factor range $\Delta K_{C P}$. The fatigue crack growth rate and the effective stress factor range has the following relational expression [5]:

$$
\begin{gathered}
\frac{d a}{d N}=C\left(\Delta K_{\text {eff }}\right)^{n} \\
\Delta K_{\text {eff }}=f(R) \Delta K_{C P}
\end{gathered}
$$

$\mathrm{C}, \mathrm{n}$ and $f(R)$ is related to the material properties and the value of stress intensity factor of combination occurrences, and the specific value can be determined through the recommendation table given in RSE-M, then $\Delta K_{\text {eff }}$ will be obtained by $\Delta K_{C P}$ according to Eq.8, and the defect growth rate $\frac{d a}{d N}$ can be obtained, supposing the value to be $\mu$, and then the propagating value of such crack under the combination occurrences $m$ and $n$ after $n_{m n}$ times can be calculated.

$$
\Delta a=\mu a n_{m n}, \Delta c=\mu c n_{m n}
$$

New crack size is as follows:

$$
a=a+\Delta a, c=c+\Delta c
$$

(5) Determination of crack size for next calculation.

Each crack tip (Point A and Point C) should be calculated, however, in each calculation, only $\Delta K$ maximum value of one tip can be found alone, if $\Delta K$ maximum value found in each combination is the value at Point $\mathrm{A}$, then $\Delta K$ value at Point $\mathrm{C}$ for this combination may not just be the maximum value. In this case, $\Delta K$ value at Point $\mathrm{C}$ can be obtained according to the combination of these two occurrences and then the crack fatigue growth at Point $\mathrm{C}$ can be obtained, where the crack size is $a+\Delta a$ and $c+\Delta c$. If the maximum value found in each combination is the value at Point $\mathrm{C}$, then $\Delta K$ at Point A can be obtained for combination of these two occurrences, finally the crack fatigue growth at Point A can be obtained, where the crack size is $a+\Delta a^{\prime}$ and 
$c+\Delta c^{\prime}$, it is obvious that their crack sizes are different and the method of $\mathrm{J}$ integral is required to assess which group of crack has the most severe effect upon the integrity of component, and the size of such most severe group of crack should be used as the initial size of next crack growth calculation [6].

(6) Occurrences considered in (5) can be eliminated once new crack sizes $a$ and $c$ have been obtained, then in the remaining combination occurrences, a new $\Delta K_{C P}$ can be obtained by repeating the calculation by (2) and (3), and then a new crack fatigue growth size can be obtained through calculation by (4) and (5).

(7) Repeating the aforesaid steps till combination of all occurrences has been analysed and the final crack growth size $a$ and $c$ will be obtained.

\section{Analysis on essential calculation factors}

\subsection{Occurrences combination}

The method of occurrences combination is used in crack fatigue growth calculation and this paper will present the method on how to use occurrences combination:

(1) Suppose that in a characterization interval there are $t$ occurrences of $a_{1}, a_{2}, \ldots, a_{\mathrm{t}}$, which are set as Array X and the occur times of each occurrence is respectively $n_{1}, n_{2}, \ldots, n_{\mathrm{t}}$, which are set as Array Y, then $K_{\mathrm{I} \max }$ and $K_{\mathrm{I} \min }$; $K_{\text {II max }}$ and $K_{\text {II min }} ; K_{\text {III max }}$ and $K_{\text {III min }}$ of each occurrence can be calculated according to Eq. 1 and 2 in Section 3; a group of $\Delta K_{\mathrm{I}}, \Delta K_{\mathrm{II}}$ and $\Delta K_{\mathrm{III}}$ can be obtained by combination of every two occurrences; a $\Delta K$ can be calculated per Eq.1, there are $C_{t}^{2} \Delta K$ in such $C_{t}^{2}$ occurrences combinations, such $C_{t}^{2}$ values will then be ranked in the order of size; suppose $\Delta K$ is maximized in combination of occurrences $m$ and $n$, which shall be counted as $\Delta K(m, n)$, and then maximum stress intensity factors $K_{\max }(m, n)$ in combination of occurrences $m$ and $n$ per Eq.2. For conservative consideration, the first calculation is always given to maximum $\Delta K(m, n)$ value in the whole process, therefore, $K_{\max }(m, n)$ shall be considered in the first calculation of crack fatigue growth, the occur times of occurrences $m$ and $n$ are respectively denoted by $n_{m}$ and $n_{n}\left(n_{m}>n_{n}\right)$, where the minimum value of such two numbers will be taken as the common occur times of the $(m, n)$ combination and denoted by $n_{m n}$, which is the times of crack fatigue growth calculation used for $(m, n)$ combination;

(2) Based on the analysis in the previous step, the occur times of occurrence $n$ has been used up within such characterization interval, then occurrence $n$ will be eliminated from Array X and $n_{n}$ be eliminated from Array Y.So $n_{m}-n_{m n}$ in occurrence $m$ has not been used up, it should be considered in the next crack fatigue growth calculation and the new arrays are denoted by $\mathrm{X} 1$ and $\mathrm{Y} 1$, and occurrences to $\mathrm{X} 1$ will be recombined to obtain $C_{t-1}^{2}$ $\Delta K$, the maximum value of which should be taken, then
$\Delta K$ value for calculation of second crack fatigue growth is available, and then the number of common occur times for second calculation of crack fatigue growth is also available.

(3) Repeating the aforesaid steps till the number of occurrences combination is zeroed.

\subsection{K-value calculation and mechanic modelling}

In the calculation of $\mathrm{K}$-value, distribution of stress $\sigma$ adjacent to defect should be calculated. The defect-free mechanic model is usually used for calculation of stress distribution of the defect zone, which is detailed as follows: Defective Model (a) can be decomposed to defect-free Model (b) and singular defective Model (c), then stress $\sigma$ equal in size and opposite in direction to stress in Model (a) has remained at the defect edge in Model (b), this portion of stress is generated by external load, while with regard to singular defective Model (c) where the stress is an internal one equal in size and opposite in direction, therefore, the internal stress distribution of component calculated using Model (b) is available for analysis of stress distribution adjacent to the defect zone [7].

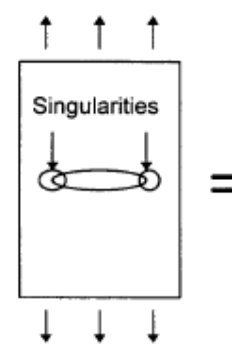

(a)

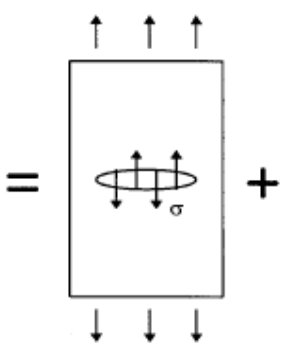

(b)

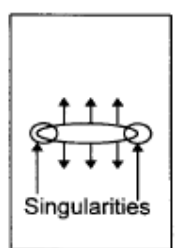

(c)
Figure 2. Equivalent analysis model for stress calculation.

Such components in the nuclear plant as the reactor pressure vessel, steam generator and pipelines are all axisymmetric models; therefore, $2 \mathrm{D}$ models are used to stress distribution calculation in the finite element software.

The 2D mechanic model of steam generator tube plate is shown in Fig. 3, the wall thickness of Segment $\mathrm{AB}$ is $t$, supposing $x$ is the distance to Point $\mathrm{A}$ in Segment $\mathrm{AB}$, and then according to RSE-M Rules, stress at the point with the distance equivalent to $x$ from internal surface can be calculated by the following formula:

$$
\sigma(x)=\left[\begin{array}{l}
\sigma_{0}+\sigma_{1} \cdot\left(\frac{x}{L}\right)+\sigma_{2} \cdot\left(\frac{x}{L}\right)^{2} \\
+\sigma_{3} \cdot\left(\frac{x}{L}\right)^{3}+\sigma_{4} \cdot\left(\frac{x}{L}\right)^{4}
\end{array}\right]
$$

Taking stresses at the points where their distances to the external surface are respectively equivalent to $1 / 5 \mathrm{~L}$, $2 / 5 L, 3 / 5 L, 4 / 5 L$ and $L$, which can be directly obtained from the calculated result by finite element software, then establishing 5 equations per Eq.11, and then can obtain 
Coefficients $\sigma_{0}, \sigma_{1}, \sigma_{2}, \sigma_{3}$ and $\sigma_{4}$. Thus, $K_{\mathrm{I}}$ can be obtained per Formula Eq.12.

$$
K_{\mathrm{I}}=\sqrt{\pi a}\left[\begin{array}{l}
\sigma_{0} \cdot i_{0}+\sigma_{1} \cdot i_{1} \cdot\left(\frac{a}{L}\right)+\sigma_{2} \cdot i_{2} \cdot\left(\frac{a}{L}\right)^{2} \\
+\sigma_{3} \cdot i_{3} \cdot\left(\frac{a}{L}\right)^{3}+\sigma_{4} \cdot i_{4} \cdot\left(\frac{a}{L}\right)^{4}
\end{array}\right]
$$

where $i_{0}, i_{1}, i_{2}, i_{3}$ and $i_{4}$ are influence coefficients, which can be acquired by RSE-M Rules, $a$ is the height of the crack and $t$ is the wall thickness.

$K_{\mathrm{II}}$ and $K_{\mathrm{III}}$ are calculated as follows:

$$
\begin{aligned}
& K_{\mathrm{II}}=\sqrt{\pi a}\left[\tau_{0} \cdot i_{0}+\tau_{1} \cdot i_{1} \cdot \frac{a}{\mathrm{t}}\right] \\
& K_{\mathrm{III}}=\sqrt{\pi a}\left[\tau_{0} \cdot i_{0}+\tau_{1} \cdot i_{1} \cdot \frac{a}{\mathrm{t}}\right]
\end{aligned}
$$

Once Stress $\sigma$ and stress intensity factors $K_{\mathrm{I}}, K_{\mathrm{II}}$ and $K_{\text {III }}$ have been calculated, other parameters can be calculated by formulas in Section 3 .

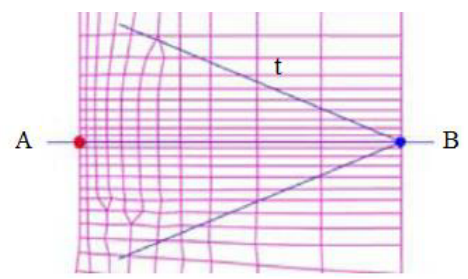

Figure 3. Diagram of 2D Mechanic Calculation Model at the Tube Plate of Steam Generator.

\subsection{Option of characterization interval}

The option of characterization interval has a direct effect upon the calculation of fatigue crack growth. The longer the characterization interval is, the smaller the final crack size is. This is because that the influence of the occur times of these occurrences themselves to $\mathrm{K}$ calculation has not been considered and thus no correction is made to $\mathrm{K}$ value with one characterization. Only the shorter the time interval is, the fewer the occurrences combination is, and such crack growth calculated in this interval would be taken into consideration during $\mathrm{K}$ calculation for the next calculation. So the shorter the time interval option is, the higher the frequency of $\mathrm{K}$ value correction is, then the calculation would be more precise.

The upper limit of characterization interval recommended in RSE-M Rules is 5 years and the lower limit is 1 year. Considering the refuelling period of most nuclear plants in China is 18 months, so from the perspective of in-service surveillance, the lower limit of interval is recommended to be 18 months.

Specific surveillance will be performed on any key components with defects during each outage, so during the calculation, the crack size shall be corrected according to the inspection result by NDT.

\section{Crack fatigue growth calculation example}

One calculation example has been given below to demonstrate this method. Suppose that there was an emerging crack whose $a=5 \mathrm{~mm}$ and $2 c=50 \mathrm{~mm}$ on the inner surface of dissimilar weld of RPV outlet nozzle to the safe end. The operation conditions are: pressure $P c=175 \mathrm{MPa}$, temperature $T c=351^{\circ} \mathrm{C}$ and the thickness of the weld is $85.5 \mathrm{~mm}$ and the material is Alloy 52.We select 6 occurrence(see Table 1 below) from the whole life time occurrences which the total number is 17 to simplify the calculation work [8].

Table 1. List of Occurrence

\begin{tabular}{|c|c|c|}
\hline Occurrence & Occurrence description & Number \\
\hline 1 & $\begin{array}{c}\text { Complete plant start-up from } \\
\text { cold shutdown to full load, with } \\
\text { RPV open at cold shutdown }\end{array}$ & 120 \\
\hline 2 & $\begin{array}{c}\text { Complete plant start-up from } \\
\text { cold shutdown to full load, with } \\
\text { RPV closed at cold shutdown }\end{array}$ & 120 \\
\hline 3 & $\begin{array}{c}\text { Complete plant shutdown from } \\
\text { full load to cold shutdown, with } \\
\text { RPV open }\end{array}$ & 120 \\
\hline 4 & $\begin{array}{c}\text { Complete plant shutdown from } \\
\text { full load to cold shutdown, with } \\
\text { RPV closed }\end{array}$ & 85 \\
\hline 5 & $\begin{array}{c}\text { Unscheduled fluctuations } \\
\text { between hot and cold shutdown }\end{array}$ & 10 \\
\hline 6 & $\begin{array}{c}\text { Hydraulic tests after } \\
\text { commissioning }\end{array}$ & 7 \\
\hline
\end{tabular}

Then the mechanic model will be built in ANSYS 11 to calculate the stress intensity factors $K_{\mathrm{I}}, K_{\mathrm{II}}$ and $K_{\mathrm{III}}$ of crack Tip C while not Tip A to simplify the calculation work. The $2 \mathrm{D}$ model is given in Figure 4.After calculating these intensity factors, according to Section 3 and 4; we use the occurrence combination to find the maximum stress intensity factors $K_{\max }(m, n)$, and then $\Delta K_{C P}$ can be defined. According to RSE-M, we defined the crack fatigue growth rate calculation formula as below. So the first crack fatigue growth has been calculated used Eq. 9 and 10. Then the second occurrence combination can be defined and the crack fatigue growth also can be calculated until the number of occurrence has all been used up.

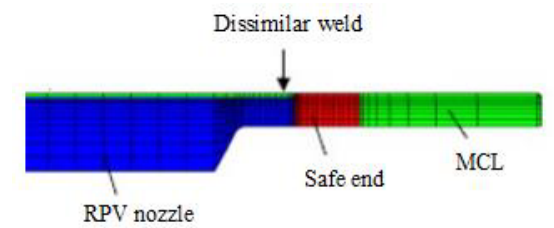

Figure 4. 2D Model of Dissimilar Weld of RPV Nozzle.

$$
\begin{gathered}
\frac{d a}{d N}=1.032 \times 10^{-12}\left(\Delta K_{e f f}\right)^{4.166} \\
\Delta K_{e f f}=f(R) \Delta K_{C P}
\end{gathered}
$$




$$
f(R)=\frac{1}{1-\frac{R}{1.63}} \quad R=\frac{K_{\min }}{K_{\max }}
$$

After repeatedly calculating, the final crack size has been calculated (see Table 2).

Table 2. Final Crack Size

\begin{tabular}{|c|c|c|c|c|}
\hline $\begin{array}{c}\text { RPV outlet } \\
\text { nozzle }\end{array}$ & $\begin{array}{c}\Delta a \\
(\mathrm{~mm})\end{array}$ & $\begin{array}{c}a+\Delta a \\
(\mathrm{~mm})\end{array}$ & $\begin{array}{c}\Delta c \\
(\mathrm{~mm})\end{array}$ & $\begin{array}{c}c+\Delta c \\
(\mathrm{~mm})\end{array}$ \\
\hline $\begin{array}{c}\text { Dissimilar } \\
\text { weld }\end{array}$ & 0.6 & 5.6 & 0.3 & 50.3 \\
\hline
\end{tabular}

\section{Conclusions}

According to the in-depth analysis on key formulas, essential factors such as occurrences combination, Kvalue calculation and mechanic modelling, option of characterization interval, and the calculation example of dissimilar weld of RPV outlet nozzle to the safe end has demonstrated a good expectation, the method of crack fatigue growth calculation recommended by RSE-M could be well used to predict the crack propagating trend of nuclear power components during ISI to assess the structure integrity. Meanwhile, this method could also be used to calculate the critical crack size to define the acceptance criteria with associated safety factors for new material applied in the third generation nuclear reactors.

\section{References}

1. RSE-M, In-Service Inspection Rules for Mechanical Components of PWR Nuclear Islands, AFCEN, 701745, (2015).

2. ASME BPVC XI, Rules for Inservice Inspection of Nuclear Power Plant Components, ASME, 437-465, (2007).

3. V. Kumar, M.D.German, An Engineering Approach for Elastic-plastic Fracture Analysis, EPRI, Palo Alto, 118-126, (1981).

4. Jin Cheng, Shushan Zhao, Fracture Mechnicals, Science Press of China, Beijing, 145, (2006).

5. H.O.Masden, S.Krenk, N.C.Lind, Methods of Structural Safety, Dover Publications, New York, 65-85, (2006).

6. J. Payne, G. Welsh and J. Robert, Int J Fatigue 32, 247 (2010).

7. Brok D, Elementary Engineering Fracture Mechanics, Noordhoff International Publishing, Leyden, 98 116, (1974).

8. Albahary Benoit, EPR ${ }^{T M}$-Assessment of Fatigue Risk in Most Sensitive Areas, Areva NP, Paris, 124-139, (2013). 